\title{
Construction and expression of a lentivirus expression vector carrying the VEGF165-EGFP fusion gene in breast cancer MCF-7 cells
}

\author{
MINNA LUO ${ }^{1}$, HUAN HUANG $^{2}$, LEI HOU $^{3}$, SHAN SHAO $^{1}$, SHANGKE HUANG $^{1}$ and XINHAN ZHAO ${ }^{1}$ \\ ${ }^{1}$ Department of Oncology, The First Affiliated Hospital of Xi'an Jiaotong University, Xi'an, Shaanxi 710061; \\ ${ }^{2}$ Department of Radiology, The Second Affiliated Hospital of Xi'an Jiaotong University, Xi'an, Shaanxi 710004; \\ ${ }^{3}$ Department of Oncology, Shaanxi Provincial People's Hospital, Xi'an, Shaanxi 710068, P.R. China
}

Received May 6, 2015; Accepted July 26, 2016

DOI: $10.3892 / 01.2017 .5601$

\begin{abstract}
Vascular endothelial growth factor (VEGF)165 is one of the most abundant and potent angiogenic factors in both physiological and pathological conditions. However, the function and mechanism of VEGF165 in tumors and their environment remain to be elucidated. In the present study, a lentivirus vector (LV) that contained the VEGF165-enhanced green fluorescent protein (EGFP) fusion gene was constructed and transfected into the human breast cancer cell line MCF-7. Following transfection, the expression of VEGF165 in MCF-7 cells was detected by reverse transcription-quantitative polymerase chain reaction (RT-qPCR) and western blotting. Further cellular localization of VEGF165 was observed through fluorescence microscopy. The titer of the recombinant lentivirus was $5.44 \times 10^{7} \mathrm{TU} / \mathrm{ml}$ in the LV-VEGF165-EGFP group and 5.00 $\times 10^{8} \mathrm{TU} / \mathrm{ml}$ in the LV-EGFP negative control group. RT-qPCR and western blotting demonstrated that the expression of VEGF165 was significantly increased in the LV-VEGF165-EGFP group compared with the control group. The present study lays the foundation for in vitro and in vivo studies on tumor cell derived-VEGF165. Furthermore, the present fusion gene expression vector may provide a potential approach for gene therapy treatment of cancer and other diseases that require regulation of angiogenesis.
\end{abstract}

\section{Introduction}

Tumors and metastases usually arise as small avascular masses that subsequently induce neovascularization in order to acquire nutrients for further growth and metastatic spread (1-3).

Correspondence to: Professor Xinhan Zhao, Department of Oncology, The First Affiliated Hospital of Xi'an Jiaotong University, 277 Yanta Western Road, Xi'an, Shaanxi 710061, P.R. China E-mail: zhaoxinhanprof@163.com

Key words: breast cancer, lentivirus vector, VEGF165-EGFP fusion gene, cellular localization
This angiogenic switch is induced by several factors such as vascular endothelial growth factor (VEGF), which is secreted by tumor cells (4). VEGF was originally identified and isolated as an endothelial cell-specific mitogen that is able to induce physiological and pathological angiogenesis $(5,6)$. Alternative splicing of VEGF leads to several different isoforms, which are differentially expressed in various tumor types and have distinct functions in tumor blood vessel formation (7). The angiogenic isoforms of VEGF are known as VEGF121, VEGF145, VEGF165 and VEGF189 in humans (8). VEGF165 is one of the most abundant and potent angiogenic agents among all the VEGF isoforms (9). Cellular responses to VEGF165 are mediated by two high-affinity type III tyrosine kinase receptors, VEGF receptor (VEGF-R)2 (also known as kinase insert domain receptor and fetal liver kinase 1) and VEGF-R1 (also known as Fms-related tyrosine kinase 1), and two receptors of the semaphorin receptor family, neuropilin-1 and neuropilin-2 (10). In addition to its functions in endothelial cells, the role of VEGF in tumor cells is currently an emerging area of importance; therefore, an increasing number of studies have focused on the biology of VEGF in tumor cells (11-13).

Vector-mediated gene expression is one of the most important tools for studying gene functions in vitro and in vivo (14). To date, several viral vectors have been applied in laboratory experiments or clinical trials and have achieved good results (15-17). In the majority of cases, the efficiency of gene transfer represents the most relevant obstacle, since it limits the success of gene overexpression or gene silencing (18). Compared with non-viral methods, viruses are highly-evolved, natural delivery agents for genetic materials $(16,19)$. In addition to their remarkable transduction efficiency, previous laboratory experiments have suggested that lentivirus vectors (LVs) exhibit low immunogenicity and durable expression (14). Furthermore, one of the most striking characteristics distinguishing the lentivirus genus from gammaretroviruses is their ability to infect non-replicating cells (20). These advantages make lentiviruses a powerful tool in the studies of gene functions.

The present study intended to construct a recombinant LV containing the VEGF165-enhanced green fluorescent protein (EGFP) fusion gene, and investigated the feasibility of using LV to express the fused VEGF165-EGFP gene in the breast cancer 
cell line MCF-7. The present study lays the foundation for future in vitro and in vivo studies on tumor cell derived-VEGF.

\section{Materials and methods}

Cell lines and cell culture. The breast carcinoma cell line MCF-7 and the human embryonic kidney epithelial cell line 293T were obtained from Shanghai Cell Bank, Chinese Academy of Sciences (Shanghai, China). All cells were cultured in Dulbecco's modified Eagle medium (DMEM; Invitrogen; Thermo Fisher Scientific, Inc., Waltham, MA, USA) supplemented with $10 \%$ fetal bovine serum (FBS; Gibco; Thermo Fisher Scientific, Inc.) and 1\% penicillin-streptomycin, and were maintained at $37^{\circ} \mathrm{C}$ under $5 \% \mathrm{CO}_{2}$. Cells used in the experiments were in logarithmic growth phase.

Construction and sequencing of pLVX-VEGF165-EGFP$3 F L A G$ recombinant plasmid expression vector. The VEGF165 (NM_001171626) coding region was amplified by polymerase chain reaction (PCR) using the PrimeScript ${ }^{\mathrm{TM}}$ RT-PCR kit (Takara Biotechnology Co., Ltd., Dalian, China) and the following cycling conditions: Pre-denaturation at $98^{\circ} \mathrm{C}$ for $3 \mathrm{~min} ; 30$ cycles at $94^{\circ} \mathrm{C}$ for $10 \mathrm{sec}, 55^{\circ} \mathrm{C}$ for $15 \mathrm{sec}$ and $72^{\circ} \mathrm{C}$ for $1 \mathrm{~min}$; and $72^{\circ} \mathrm{C}$ for a final $10 \mathrm{~min}$. The VEGF165 fragment DNA was extracted and purified using the QIAquick Gel Extraction kit and the QIAquick PCR Purification kit, respectively (Qiagen GmbH, Hilden, Germany). The purified VEGF165 fragment and the pLVX-EGFP-3FLAG plasmid (Shanghai Sbo-bio Biotechnology, Shanghai, China) (Fig. 1) were digested separately using EcoRI restriction endonuclease. Upon digestion, the mixture was incubated at $65^{\circ} \mathrm{C}$ for $10 \mathrm{~min}$ to stop the reaction, after which the products were separated by $1.5 \%$ agarose gel electrophoresis. The bands were visualized using a gel scanning analysis system (ChemiDoc MP imaging system; Bio-Rad Laboratories, Inc., Hercules, CA, USA). Then, the target fragment of about $7.7 \mathrm{~kb}$ in size was recycled by $1 \%$ agarose gel electrophoresis.

Using the In-Fusion ${ }^{\circledR}$ HD Cloning kit (Clontech Laboratories, Inc., Mountainview, CA, USA), the VEGF165 fragment was ligated into the pLVX-EGFP-3FLAG expression vector, which had been digested with EcoRI. Two control groups were used, including a blank control and a negative control group. Next, the recombinant plasmid vector was transformed into Escherichia coli DH5 $\alpha$ competent bacteria (Takara Biotechnology Co., Ltd.) according to the manufacturer's protocol, and Luria broth (LB)-agar plates containing $100 \mu \mathrm{g} / \mathrm{ml}$ ampicillin were employed to select the positive clones. Upon transformation, the positive clones were identified by PCR using the PrimeScript ${ }^{\mathrm{TM}}$ RT-PCR kit and the following cycling conditions: Pre-denaturation at $94^{\circ} \mathrm{C}$ for $5 \mathrm{~min} ; 30$ cycles at $94^{\circ} \mathrm{C}$ for $30 \mathrm{sec}, 55^{\circ} \mathrm{C}$ for $30 \mathrm{sec}$ and $72^{\circ} \mathrm{C}$ for $1 \mathrm{~min}$; and $72^{\circ} \mathrm{C}$ for $10 \mathrm{~min}$.

The $E$. coli clones were cultured in $\mathrm{LB}$ medium at $37^{\circ} \mathrm{C}$, and the plasmid was extracted using the QIAprep Spin Miniprep kit according to the manufacturer's protocol (Qiagen $\mathrm{GmbH}$ ). The VEGF165 gene was then amplified by PCR using the Prime Script ${ }^{\mathrm{TM}}$ RT-PCR kit to identify the correct recombinant plasmids. The cycling conditions were: Pre-denaturation at $94^{\circ} \mathrm{C}$ for $5 \mathrm{~min} ; 30$ cycles at $94^{\circ} \mathrm{C}$ for $30 \mathrm{sec}, 55^{\circ} \mathrm{C}$ for $30 \mathrm{sec}$ and $72^{\circ} \mathrm{C}$ for $1 \mathrm{~min}$; and $72^{\circ} \mathrm{C}$ for $10 \mathrm{~min}$. The CMV-F and pEGFP-N-3 primers were used to set up the PCR reaction, and their sequences are indicated in Table I. The positive bacteria colonies were inoculated into $\mathrm{LB}$ medium at $37^{\circ} \mathrm{C}$ for $16 \mathrm{~h}$, and upon adding glycerol, they were stored at $-80^{\circ} \mathrm{C}$. Aliquots (200 $\mu \mathrm{l}$ ) of each were sent for DNA sequencing by Shanghai Sbio-bio Biotechnology Co. Ltd. (Shanghai, China).

Transfection of the VEGF165 recombinant lentiviral plasmid in $293 \mathrm{~T}$ cells. At $24 \mathrm{~h}$ prior to transfection, 293T cells growing in the logarithmic phase were selected and propagated. The cell number was adjusted to $6 \times 10^{5}$ cells $/ \mathrm{ml}$ with $10 \%$ FBS DMEM. The cells were transfected at $60-70 \%$ confluence, and $2 \mathrm{~h}$ prior to transfection, the medium was changed to serum-free medium. The recombinant $p L V X-V E G F 165-E G F P-3 F L A G$ vector and three packaging components (pRSV-REV, pMDLg-pRRE and pMD2.G) DNAs (Addgene, Inc., Cambridge, MA, USA) were added into a sterile centrifuge tube and mixed with an appropriate volume of Gibco $^{\mathrm{TM}}$ Opti-MEM ${ }^{\mathrm{TM}}$ (Thermo Fisher Scientific, Inc.). The 293T cells were then co-transfected with the above DNAs using Lipofectamine 2000 according to the manufacturer's protocol (Thermo Fisher Scientific, Inc.). At $8 \mathrm{~h}$ post-transfection, the medium was replaced with complete culture medium, and the cells were continuously cultured for $48 \mathrm{~h}$. Subsequently, the supernatant containing lentivirus particles was harvested and concentrated by ultracentrifugation at $4,000 \times \mathrm{g}$ for $20 \mathrm{~min}$ at $4^{\circ} \mathrm{C}$ to obtain a high-titer lentivirus concentration. $293 \mathrm{~T}$ cells were used to measure the supernatant virus titer.

Western blot analysis of VEGF165 expression in 2937 cells. Transfected and non-transfected 293T cells were harvested, and proteins were extracted using radioimmunoprecipitation assay lysis buffer (Pioneer Biotech, Co., Ltd., Shaanxi, China) containing protease inhibitors for $30 \mathrm{~min}$ on ice, and then cleared at $25,000 \mathrm{x} g$ for $20 \mathrm{~min}$ at $4^{\circ} \mathrm{C}$. The protein concentration was determined using the Bradford assay (Sigma-Aldrich; Merck Millipore, Darmstadt, Germany). Upon denaturation by heating at $100^{\circ} \mathrm{C}$ for $5-10 \mathrm{~min}$, equivalent amounts of total cellular protein were subjected to reducing sodium dodecyl sulfate-polyacrylamide gel electrophoresis (8-12\%), followed by blotting on a polyvinylidene difluoride membrane. After blocking in 5\% non-fat dry milk for $2 \mathrm{~h}$, membranes were incubated with the mouse anti-VEGF monoclonal antibody (ab1316, 1:100; Abcam, Cambridge, UK), the mouse anti-GFP polyclonal antibody (sc-9996, 1:3,000; Santa Cruz Biotechnology, Inc., Dallas, TX, USA) and the mouse anti- $\beta$-actin monoclonal antibody (sc-69879, 1:1,000; Santa Cruz Biotechnology, Inc.) overnight at $4^{\circ} \mathrm{C}$. The membranes were then washed with TBST three times for $5 \mathrm{~min}$ each, and incubated with a horseradish peroxidase (HRP)-conjugated secondary antibody (Santa Cruz Biotechnology, Inc.) for $2 \mathrm{~h}$ at room temperature and visualized by enhanced chemiluminescence (GE Healthcare Life Sciences, Chalfont, UK). Images were documented by a scanner, quantified using Pro-Plus 6.0 software (Media Cybernetics, Inc., Rockville, MD, USA) and analyzed using ImageJ 1.49 software (National Institutes of Health, Bethesda, MD, USA).

Detection of recombinant lentiviral titer by quantitative (q)PCR. Viral titers were measured by qPCR, using $\beta$-actin as an internal control. The quantification cycle $(\mathrm{Cq})$ value was defined as the number of cycles when the fluorescent signal 
Table I. Primers used for VEGF165 gene fragment amplification and RT-qPCR.

\begin{tabular}{ll} 
Primer & \multicolumn{1}{c}{ Sequence } \\
\hline VEGF165-EcoRI-Forward & 5'-CTCAAGCTTCGAATTCGCCACCATGAACTTTCTGCTGTCTTGG-3' \\
VEGF165-EcoRI-Reverse & 5'-CATGGTGGCGAATTCCCGCCTCGGCTTGTCACA-3' \\
CMV-F & 5'-CGCAAATGGGCGGTAGGCGTG-3' \\
pEGFP-N3 & 5'-CGTCGCCGTCCAGCTCGACCAG-3' \\
EGFP-Forward & 5'-CCTTTCCGGGACTTTCGCTTT-3' \\
EGFP-Reverse & 5'-GCAGAATCCAGGTGGCAACA-3' \\
$\beta$-actin-Forward & 5'-AAGAGAGGCATCCTCACCCT-3' \\
$\beta$-actin-Reverse & 5'-TACATGGCTGGGGTGTTGAA-3' \\
$\beta$-actin-forward & 5'-GGAGATTACTGCCCTGGCTCCTA-3' \\
$\beta$-actin-Reverse & 5'-GACTCATCGTACTCCTGCTTGCTG-3' \\
VEGF165-Forward $^{\mathrm{b}}$ & 5'-ATCTTCAAGCCATCCTGTGTGC-3' \\
VEGF165-Reverse $^{\text {a }}$ & 5'-CAAGGCCCACAGGGATTTTC-3'
\end{tabular}

${ }^{a} \beta$-actin was used to detect the virus titer in 293T cells. ${ }^{b} \beta$-actin was used in RT-qPCR analysis of VEGF165 expression in MCF-7 cells. VEGF, vascular endothelial growth factor; EGFP, enhanced green fluorescent protein; RT-qPCR, reverse transcription-quantitative polymerase chain reaction.

reached a specified threshold, as described previously (21). The sequences of the primers used are presented in Table I.

Briefly, 293T cells $\left(1 \times 10^{5}\right)$ were placed into each well of a 6 -well plate for $24 \mathrm{~h}$ culture, and $10 \mu \mathrm{l}$ of a virus stock solution was added into an Eppendorf tube containing $90 \mu \mathrm{l}$ of cell culture medium, mixed and diluted 10X. Three different concentrations were added separately to the wells where the 293T cells were cultured. After 48-h culture, complete culture medium was added, and 4 days later, the fluorescence expression was examined. Total RNA was extracted from the cells with TRIzol (Invitrogen; Thermo Fisher Scientific, Inc.). The reverse transcription (RT) reaction was then performed for complementary (c)DNA synthesis using the PrimeScript First Strand cDNA Synthesis kit (Takara Biotechnology Co., Ltd.). qPCR was performed using the SYBR Green PCR kit (Takara Biotechnology Co., Ltd.) and the following cycling conditions: Pre-denaturation at $95^{\circ} \mathrm{C}$ for $15 \mathrm{sec}$, followed by 40 cycles at $95^{\circ} \mathrm{C}$ for $5 \mathrm{sec}, 60^{\circ} \mathrm{C}$ for $30 \mathrm{sec}$ and $60^{\circ} \mathrm{C}$ for $30 \mathrm{sec}$.

Lentivirus infection of $M C F-7$ cells. Concentrated lentivirus solutions of LV-EGFP and LV-VEGF165-EGFP were added separately into two wells of cultured MCF-7 cells once the cells reached $40 \%$ confluence. Enhanced infection solution (Shanghai Sbo-bio Biotechnology) was then added to reach a total incubation volume of $2 \mathrm{ml}$. After $12 \mathrm{~h}$ of incubation, the cell culture medium was changed, and $72 \mathrm{~h}$ later, cells expressing EGFP were imaged under an inverted fluorescence microscope (Eclipse Ti-E; Nikon Corporation, Tokyo, Japan). Images were processed using NIS-Elements Basic Research Imaging Software (Nikon Corporation).

RT-qPCR and western blot analysis of VEGF165 expression in vitro. Stably transfected MCF-7 cells were selected by limiting dilution, and the expression levels of VEGF165 in the cells were measured by RT-qPCR and western blotting. Total RNA and total protein were extracted from MCF-7 cells transfected with LV-EGFP and LV-VEGF165-EGFP. Western

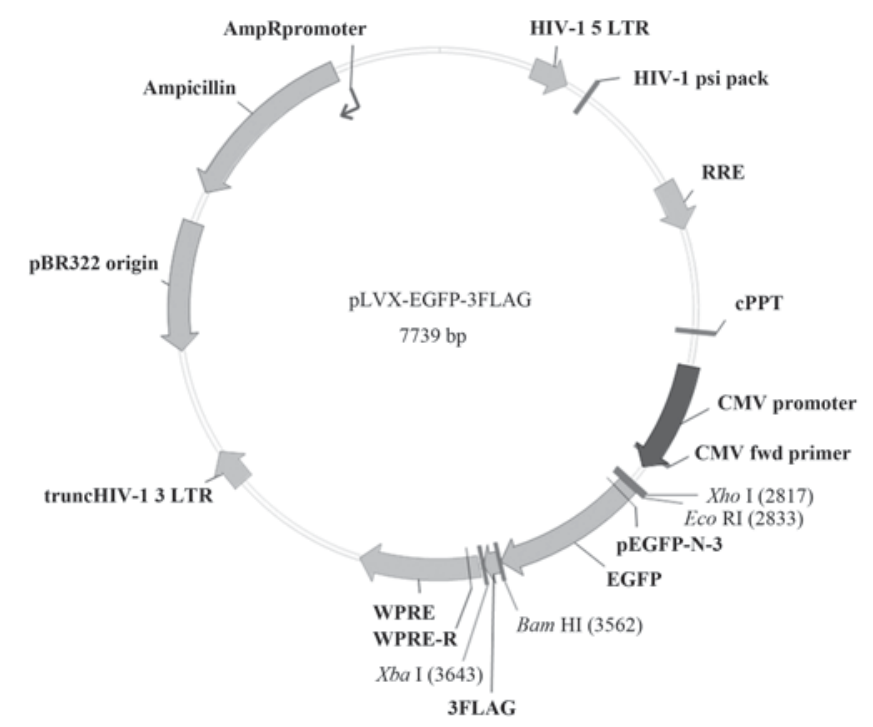

Figure 1. Structure of the eukaryotic expression vector pLVX-EGFP-3FLAG. Image adapted from Shanghai Sbio-bio Biotechnology Co. Ltd. LV, lentivirus vector; EGFP, enhanced green fluorescent protein.

blotting was performed as described above, and the target proteins were hybridized with mouse anti-VEGF monoclonal antibody (1:200, Abcam), mouse anti-GFP (1:1,000, ProteinTech Group, Inc., Chicago, IL, USA), mouse anti- $\beta$-actin antibody (1:5,000, ProteinTech Group, Inc.) and HRP-conjugated goat anti-mouse immunoglobulin G (1:10,000, CWBIO, Beijing, China). Enhanced chemiluminescent substrates (SuperSignal $^{\text {TM }}$ West Femto Maximum Sensitivity Substrate; Pierce; Thermo Fisher Scientific, Inc.) were used to detect the signals of targeted proteins.

Statistical analyses. All statistical analyses were performed using SPSS 16.0 software (SPSS, Inc., Chicago, IL, USA). Data are expressed as the mean \pm standard error of the mean. One way analysis of variance was used for comparisons among 
three groups. $\mathrm{P}<0.05$ was considered to indicate a statistically significant difference.

\section{Results}

pLVX-VEGF165-EGFP-3FLAG plasmid construction. The expected PCR product size of $611 \mathrm{bp}$ for the VEGF165 coding sequence was obtained (Fig. 2A), and the PCR products of VEGF165 and the pLVX-EGFP-3FLAG plasmid were then digested separately with $E c o$ RI (Fig. 2B), followed by construction of the pLVX-VEGF-EGFP-3FLAG vector via In-Fusion ${ }^{\mathrm{TM}}$ Enzyme ligation (Clontech Laboratories, Inc.). Competent E. coli $\mathrm{DH} 5 \alpha$ cells were transformed with the above plasmid, and three positive bacteria clones containing a fusion gene composed of the VEGF165 coding sequence and a fragment of the EGFP gene sequence were identified using PCR, which detected a product of $837 \mathrm{bp}$ in size (Fig. 2C). The sequence of the recombinant plasmid pLVX-VEGF165-EGFP-3FLAG was confirmed by sequencing, and the VEGF165 sequence length was $611 \mathrm{bp}$. This sequence was consistent with that of the human VEGF165 gene published in the GenBank ${ }^{\circledR}$ database (http://www.ncbi.nlm.nih.gov/nuccore/NM_001171626.1), with the exception of a synonymous mutation from ACC to ACA, which is not expected to influence the protein synthesis (Fig. 3A and B).

Transfection of the VEGF165 recombinant lentiviral plasmid in 2937 cells, and expression of EGFP and VEGF165. The recombinant pLVX-VEGF165-EGFP-3FLAG vector and three packaging components, pRSV-REV, pMDLg-pRRE and pMD2.G DNAs, were co-transfected in 293T cells. The expression of EGFP and VEGF165 was detected by western blotting. When incubated with an anti-VEGF antibody, the VEGF165 fusion protein at a specific band size of $53 \mathrm{kDa}$ was uniquely observed in VEGF165 recombinant lentiviraltransfected 293T cells, while expression of the EGFP fusion protein $(53 \mathrm{kDa})$ was observed in both the LV-VEGF165-EGFP group and the LV-EGFP group if incubated with an anti-GFP antibody. This band was consistent with the expected size of the VEGF165-EGFP fusion protein, indicating that the VEGF165 gene fused with the EGFP gene, and both could be co-expressed in $293 \mathrm{~T}$ cells. Furthermore, the aforementioned band was not detected in non-transfected cells (Fig. 3C).

VEGF165 recombinant lentiviral packaging and determination of virus titer. To examine the virus titer of each group, qPCR was performed. The difference of $\mathrm{Cq}$ values was compared between the control and the test group in order to determine the titer concentration. $\mathrm{Cq}$ values $>2.0$ were considered to be significantly different. The titer of recombinant lentivirus was $5.44 \times 10^{7} \mathrm{TU} / \mathrm{ml}$ in the LV-VEGF165-EGFP group and $5.00 \times 10^{8} \mathrm{TU} / \mathrm{ml}$ in the LV-EGFP negative control group, as calculated according to the following formula: Virus titer $(\mathrm{IU} / \mathrm{ml})=(\mathrm{CxNxDx} 1,000) / \mathrm{V}$, where $\mathrm{V}$ is the volume of the diluted virus solution in $\mu \mathrm{l}, \mathrm{C}$ is the number of virus copies integrated in each cell genome, $\mathrm{N}$ is the cell number and $\mathrm{D}$ is the dilution of the virus solution (Table II).

Observation of cellular localization of VEGF165 expression in MCF-7 cells by fluorescence microscopy. After $72 \mathrm{~h}$ of
A

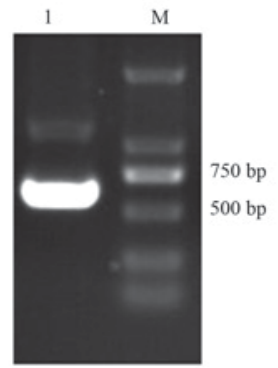

B

$7.7 \mathrm{~kb}$

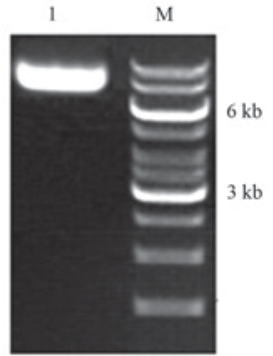

C

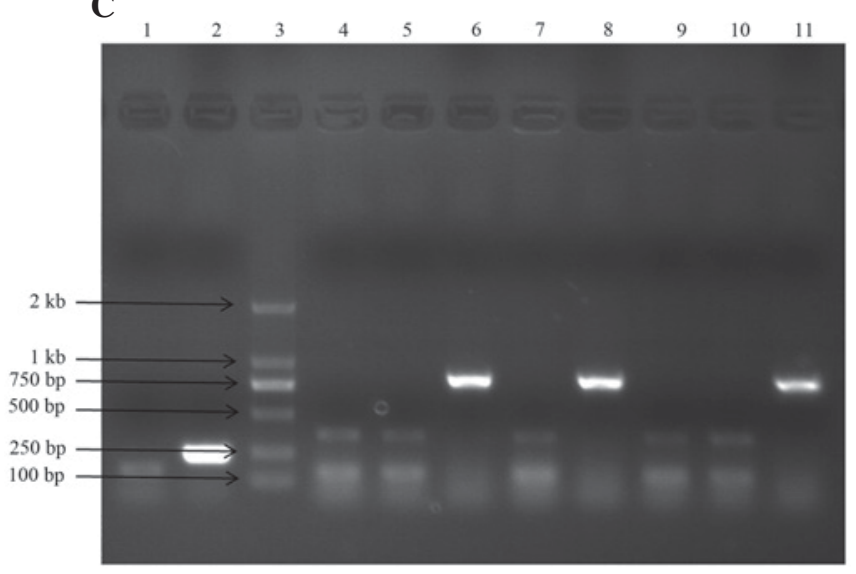

Figure 2. (A) PCR product for the VEGF165 coding sequence (611 bp). (B) Linearized pLVX-EGFP-3FLAG plasmid upon digestion with EcoRI (7.7 kb). (C) The pLVX-VEGF165-EGFP-3FLAG recombinant expression vector was identified in bacterial clones by PCR. Lane 1, blank control group; lane 2, negative control group (LV-EGFP vector, 252 bp); lane 3, DL2000 DNA Marker; lanes 4-11: eight pLVX-VEGF165-EGFP-3FLAG-transformed clones, of which, clones 6, 8 and 11 were positive clones (837-bp band). M, marker; LV, lentivirus vector; VEGF, vascular endothelial growth factor; EGFP, enhanced green fluorescent protein; PCR, polymerase chain reaction.

transfection, MCF-7 cells were observed under a fluorescence microscope to assess the expression of EGFP. Both LV-EGFP and LV-VEGF165-EGFP groups demonstrated transfection efficiencies $>80 \%$. As represented in Fig. 4A-F, EGFP was highly expressed in MCF-7 cells transfected with LV. By contrast, no EGFP signal was detected in non-transfected cells. Fluorescence microscopy was used to observe the cellular localization of VEGF165 in MCF-7 cells. As represented in Fig. 4G and H, VEGF165-EGFP was mainly expressed in the cell membrane and nucleus of MCF-7 cells, and certain expression was also noticed outside the cell membrane. This indicates that the VEGF165-EGFP fusion protein could be expressed and secreted normally.

Detection of the VEGF165 expression in MCF-7 cell lines by $R T-q P C R$ and western blotting. RT-qPCR and western blotting were applied to detect the RNA and protein expression levels of VEGF165-EGFP, respectively. RT-qPCR demonstrated that the expression of VEGF165 in the LV-VEGF165-EGFP group was significantly increased (relative gray value of PCR, $0.4019 \pm 0.1143$ and $0.2147 \pm 0.0965$ in the LV-VEGF165-EGFP and LV-EGFP groups, respectively; Fig. 5A and B). Similarly, compared with non-transfected cells, a characteristic band of $53 \mathrm{kDa}$ was observed by western blotting in MCF-7 cells transfected with LV-VEGF165-EGFP, whose size was consistent with that of the VEGF165-EGFP fusion protein (Fig. 5C), indicating 
Table II. Virus titers in the LV-VEGF165-EGFP and LV-EGFP groups.

\begin{tabular}{|c|c|c|c|c|c|c|}
\hline Group & $\mathrm{V}$ & $\mathrm{C}$ & $\mathrm{N}$ & $\mathrm{D}$ & Virus titer ${ }^{\mathrm{a}}$ & Average titer \\
\hline \multicolumn{7}{|c|}{ LV-VEGF165-EGFP group } \\
\hline $10 \mu 1$ & 10.0 & 0.60 & $2 \times 10^{5}$ & 1 & $1.20 \times 10^{7}$ & $5.44 \pm 4.20 \times 10^{7}$ \\
\hline $1 \mu 1$ & 1.0 & 0.28 & $2 \times 10^{5}$ & 1 & $5.54 \times 10^{7}$ & \\
\hline $10^{-1} \mu 1$ & 0.1 & 0.05 & $2 \times 10^{5}$ & 1 & $9.60 \times 10^{7}$ & \\
\hline \multicolumn{7}{|c|}{ LV-EGFP group } \\
\hline $10 \mu 1$ & 10.0 & 15.56 & $2 \times 10^{5}$ & 1 & $3.12 \times 10^{8}$ & $5.00 \pm 1.85 \times 10^{8}$ \\
\hline $1 \mu 1$ & 1.0 & 3.41 & $2 \times 10^{5}$ & 1 & $6.82 \times 10^{8}$ & \\
\hline $10^{-1} \mu 1$ & 0.1 & 0.25 & $2 \times 10^{5}$ & 1 & $5.06 \times 10^{8}$ & \\
\hline
\end{tabular}

${ }^{a}$ Virus titer $(\mathrm{IU} / \mathrm{ml})=(\mathrm{C} \times \mathrm{N} \times \mathrm{D} \times 1,000) / \mathrm{V} . \mathrm{V}$, volume of the diluted virus solution $(\mu 1) ; \mathrm{C}$, number of virus copies integrated in each cell genome; N, cell number; D, dilution of the virus solution. LV, lentivirus vector; VEGF, vascular endothelial growth factor; EGFP, enhanced green fluorescent protein.

A

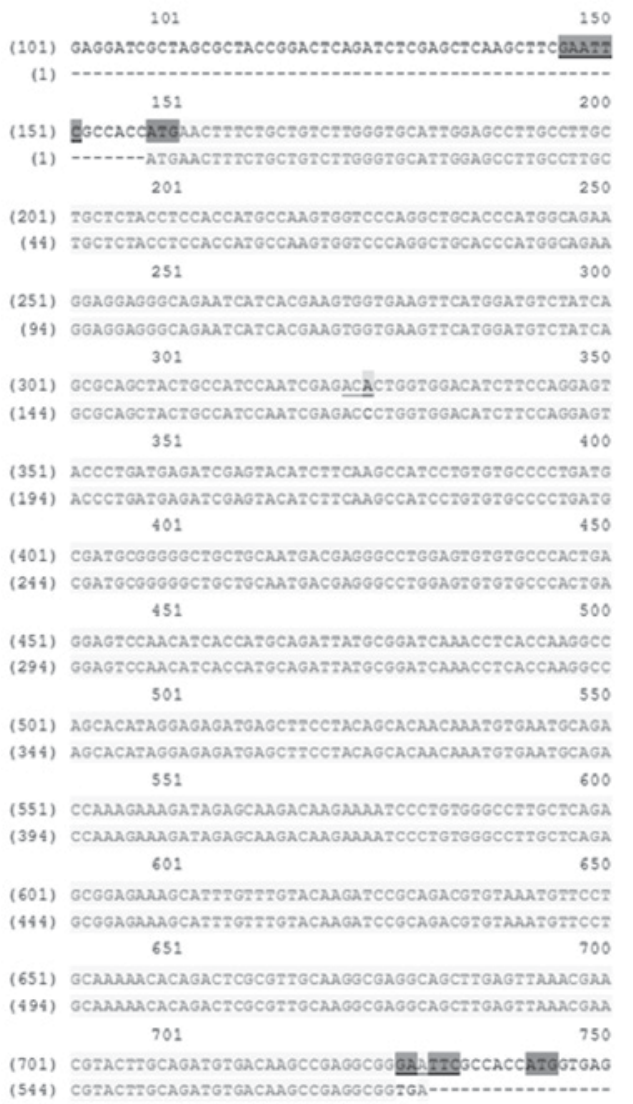

B

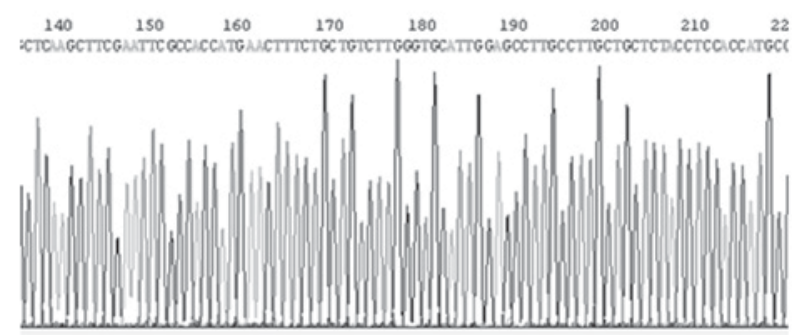

C

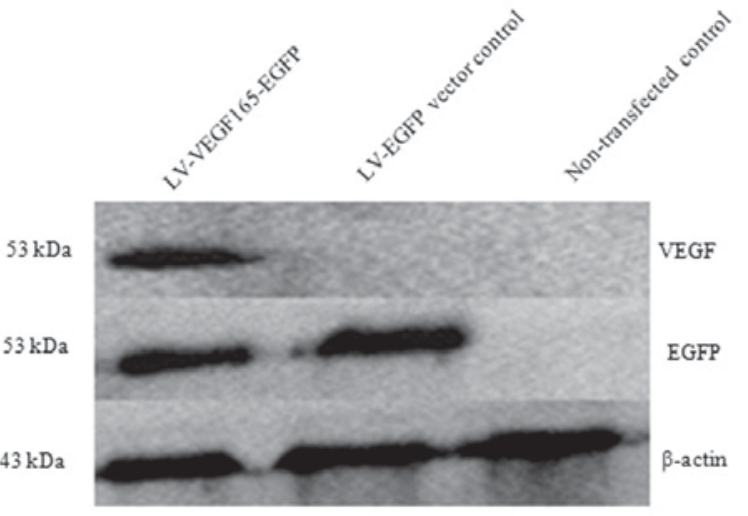

Figure 3. (A) VEGF165 gene sequencing of the pLVX-VEGF165-EGFP-3FLAG vector following the selection of positive clones. (B) Sequencing waveform for the pLVX-VEGF165-EGFP-3FLAG vector. (C) Western blot analysis at $48 \mathrm{~h}$ post-transfection of VEGF165 recombinant lentiviral plasmid in 293T cells. The molecular weight of the fused VEG165-EGFP protein was $53 \mathrm{kDa}$; therefore, EGFP and VEGF165 were both detected in the range of $53 \mathrm{kDa}$. LV, lentivirus vector; VEGF, vascular endothelial growth factor; EGFP, enhanced green fluorescent protein.

that the VEGF165-EGFP fusion gene in the recombinant LV could be expressed following transfection into MCF-7 cells.

\section{Discussion}

It has been reported that different isoforms of VEGF are detected in individual tissues; however, VEGF165 is the most abundant one in almost all human organs with the exception of the lung (22). Several studies revealed that VEGF165 was highly expressed in breast cancer tissues compared with adjacent tissues $(23,24)$. RT-qPCR experiments demonstrated that four isoforms of VEGF were expressed in human breast cancer tissues, including VEGF121, VEGF145, VEGF165 and VEGF189, being VEGF165 the most abundant one (25). In addition, previous studies demonstrated that VEGF165 fused to GFP at its C-terminus was secreted and biologically active (26). 
A

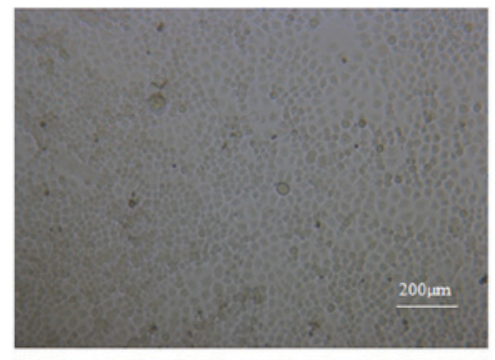

C

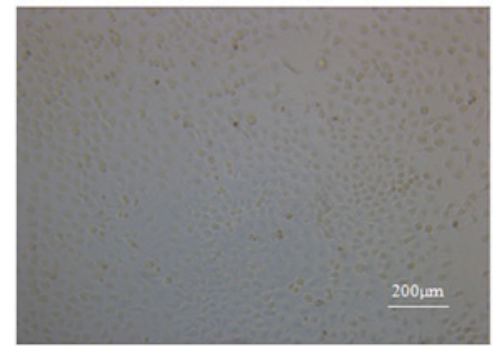

$\mathbf{E}$

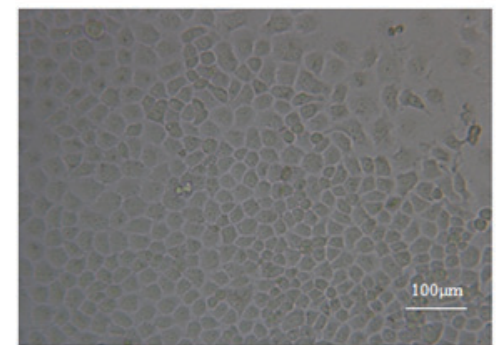

G

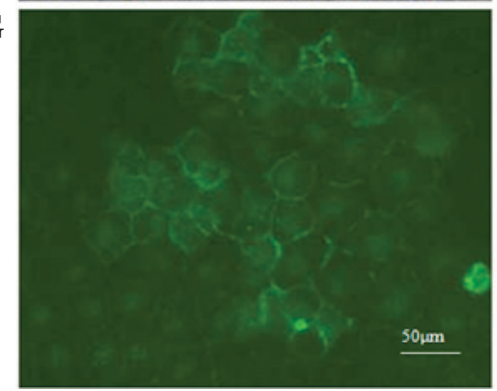

B

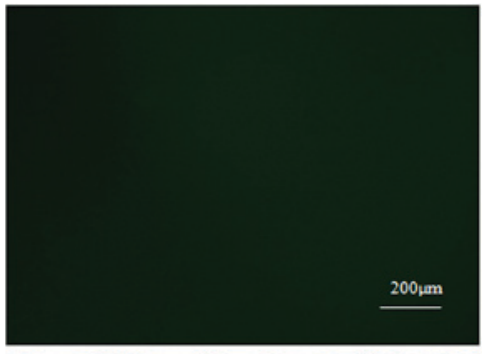

D

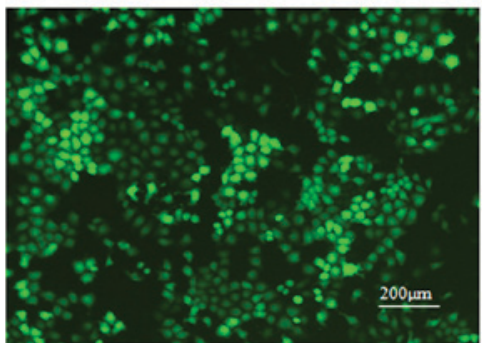

$\mathbf{F}$

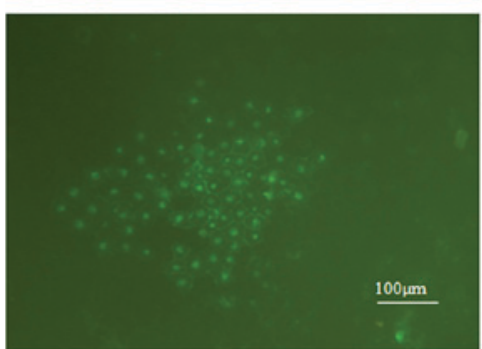

$\mathbf{H}$

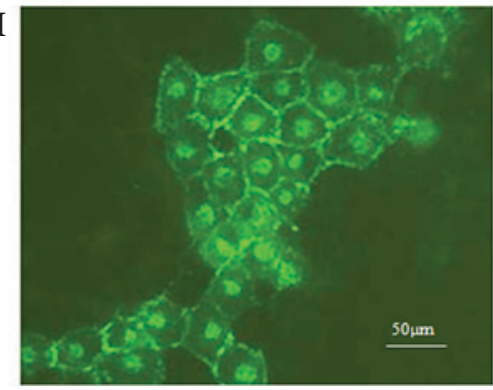

Figure 4. Expression of recombinant lentivirus carrying the VEGF165-EGFP fusion gene in MCF-7 cells after $72 \mathrm{~h}$ of transfection. Non-transfected MCF-7 cells in (A) bright-field and (B) dark-field (magnification, x100). LV-EGFP-transfected MCF-7 cells in (C) bright-field and (D) dark-field (magnification, x100). LV-VEGF165-EGFP-transfected MCF-7 cells in (E) bright-field and (F) dark-field (magnification, x200). Cells transfected with LV-EGFP and LV-VEGF165-EGFP expressed high levels of EGFP, indicating successful transfection of lentivirus in cultured MCF-7 cells. (G) LV-VEGF165-EGFP transfected MCF-7 cells in dark-field (magnification, x400). (H) Observation of cellular localization of VEGF165 expression in MCF-7 cells (magnification, x400). LV, lentivirus vector; VEGF, vascular endothelial growth factor; EGFP, enhanced green fluorescent protein.

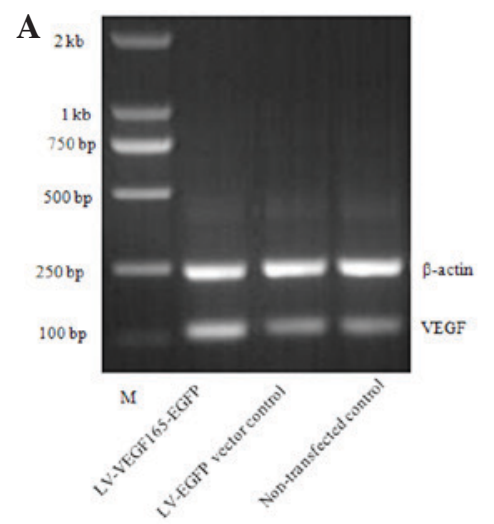

B

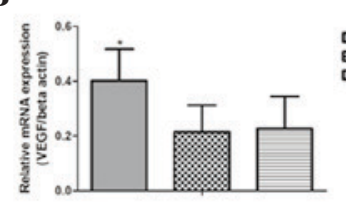

C

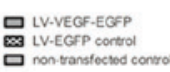
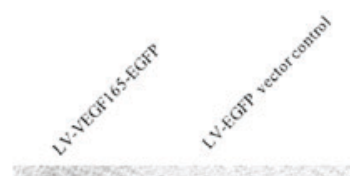

VEGF-EGFP fused protein VEGF B-actin

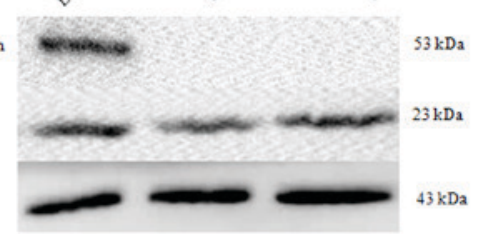

Figure 5. Detection of mRNA and protein levels of VEGF165 in MCF-7 cells following transfection. (A) Polymerase chain reaction analysis of VEGF165 mRNA levels in MCF-7 cells upon transfection. (B) Histogram of VEGF165 mRNA levels in MCF-7 cells. Compared with non-transfected cells and cells transfected with LV-EGFP, cells transfected with LV-VEGF165-EGFP contained significantly higher amounts of VEGF mRNA. Columns indicate means; bars indicate standard error. "P<0.05 vs. control. (C) Western blot analysis of VEGF165-EGFP fusion protein upon transfection. VEGF165-EGFP fusion protein could only be detected in the LV-VEGF165-EGFP group, while normal VEGF could be detected in all the three groups. LV, lentivirus vector; VEGF, vascular endothelial growth factor; EGFP, enhanced green fluorescent protein; mRNA, messenger RNA. 
Therefore, in order to elucidate the function of tumor cell derived-VEGF in breast cancer biology, VEGF165 was selected as the target gene in the present study, and the VEGF165-EGFP fusion gene expression vector was constructed.

As a newly developed technology, recombinant expression through viral vectors have been used in numerous laboratory experiments and clinical trials (27-29). There are mainly five types of viral vectors that are commonly used for recombinant expression, namely adenovirus, adeno-associated virus, herpes simplex virus, retrovirus and lentivirus (14). Due to the wide range of host cells, the ability to infect both replicating and non-replicating cells, the easy integration of the exogenous gene in the host cells, and the long-lasting and stable expression of exogenous genes, lentivirus was the most suitable type of vector for our experiments $(14,30)$. In the present study, a VEGF165-EGFP fusion gene recombinant lentiviral expression vector was first constructed. Using molecular cloning techniques such as PCR and DNA sequencing, the human VEGF165-EGFP fusion gene lentiviral expression plasmid was successfully constructed, and high-titer viral particles were obtained following plasmid transfection in packaging cells. Due to its safety and high transfection efficiency (31), a four-plasmid lentivirus packaging system was employed in the current study, including a carrier plasmid containing the VEGF165-EGFP fusion gene, a packaging plasmid pRSV-Rev encoding Rev response element (RRE), a packaging plasmid pMDLg-pRRE containing the Gag-Pol coding sequence, and a packaging plasmid pMD2.G encoding the vesicular stomatitis virus (VSV)-G envelope protein. In this packaging system, the RRE and Gag-Pol coding sequences are located in two different plasmids, which greatly reduces the possibility of autonomous viral replication $(31,32)$. Furthermore, the human immunodeficiency virus-derived Env gene was replaced by the VSV-G gene in our packaging system, which significantly increased the range of host cell types that could be infected.

The VEGF165-EGFP expression rate was $>80 \%$ in infected human breast cancer cells MCF-7. Furthermore, it was observed that VEGF165 was mainly located on the cell membrane and nucleus of MCF-7 cells by fluorescence microscopy. The constructed VEGF165 lentiviral recombinant vector will be used in future studies to evaluate the function of the target gene, following its stable transfection in tumor cell lines. This will enable a detailed examination of the mechanism of tumor cell derived-VEGF165 in tumor cells and in the tumor microenvironment. Besides, this fusion gene expression vector may also provide a potential approach for gene therapy in diseases that require regulation of angiogenesis.

\section{Acknowledgements}

The present study was supported by Specialized Research Fund for the Doctoral Program of Higher Education of China (Beijing, China; grant no. 20100201110059).

\section{References}

1. Lichtenberger BM, Tan PK, Niederleithner H, Ferrara N Petzelbauer P and Sibilia M: Autocrine VEGF signaling synergizes with EGFR in tumor cells to promote epithelial cancer development. Cell 140: 268-279, 2010.
2. Ribatti D, Nico B, Crivellato E, Roccaro AM and Vacca A: The history of the angiogenic switch concept. Leukemia 21: 44-52, 2006.

3. Roskoski R Jr: Vascular endothelial growth factor (VEGF) signaling in tumor progression. Crit Rev Oncol Hematol 62: 179-213, 2007.

4. Bergers $\mathrm{G}$ and Benjamin LE: Tumorigenesis and the angiogenic switch. Nat Rev Cancer 3: 401-410, 2003.

5. Ferrara N and Davis-Smyth T: The biology of vascular endothelial growth factor. Endocr Rev 18: 4-25, 1997.

6. Carmeliet P and Jain RK: Molecular mechanisms and clinical applications of angiogenesis. Nature 473: 298-307, 2011.

7. Mac Gabhann F and Popel AS: Systems biology of vascular endothelial growth factors. Microcirculation 15: 715-738, 2008.

8. Vempati P, Popel AS and Mac GF: Extracellular regulation of VEGF: Isoforms, proteolysis and vascular patterning. Cytokine Growth Factor Rev 25: 1-19, 2014.

9. Otrock ZK, Makarem JA and Shamseddine AI: Vascular endothelial growth factor family of ligands and receptors: Review. Blood Cells Mol Dis 38: 258-268, 2007.

10. Ferrara N, Gerber HP and LeCouter J: The biology of VEGF and its receptors. Nat Med 9: 669-676, 2003.

11. Knizetova P, Ehrmann J, Hlobilkova A, Vancova I, Kalita O, Kolar Z and Bartek J: Autocrine regulation of glioblastoma cell-cycle progression, viability and radioresistance through the VEGF-VEGFR2 (KDR) interplay. Cell Cycle 7: 2553-2561, 2008.

12. Chakraborty G, Jain S and Kundu GC: Osteopontin promotes vascular endothelial growth factor-dependent breast tumor growth and angiogenesis via autocrine and paracrine mechanisms. Cancer Res 68: 152-161, 2008.

13. Hamerlik P, Lathia JD, Rasmussen R, Wu Q, Bartkova J, Lee M, Moudry P, Bartek J Jr, Fischer W, Lukas J, et al: Autocrine VEGF-VEGFR2-Neuropilin-1 signaling promotes glioma stem-like cell viability and tumor growth. J Exp Med 209: 507-520, 2012

14. Vannucci L, Lai M, Chiuppesi F, Ceccherini-Nelli L and Pistello M: Viral vectors: A look back and ahead on gene transfer technology. New Microbiol 36: 1-22, 2013.

15. Nathwani AC, Tuddenham EGD, Rangarajan S, Rosales C, McIntosh J, Linch DC, Chowdary P, Riddell A, Pie AJ, Harrington C, et al: Adenovirus-associated virus vector-mediated gene transfer in hemophilia b. N Engl J Med 365: 2357-2365, 2011.

16. Giacca $\mathrm{M}$ and Zacchigna S: Virus-mediated gene delivery for human gene therapy. J Control Release 161: 377-388, 2012.

17. Huang S and Kamihira M: Development of hybrid viral vectors for gene therapy. Biotechnol Adv 31: 208-223, 2013.

18. Lehto T, Simonson OE, Mager I, Ezzat K, Sork H, Copolovici D, Viola JR, Zaghloul EM, Lundin P, Moreno PM, et al: A peptide-based vector for efficient gene transfer in vitro and in vivo. Mol Ther 19: 1457-1467, 2011.

19. Mingozzi $F$ and High KA: Therapeutic in vivo gene transfer for genetic disease using AAV: Progress and challenges. Nat Rev Genet 12: 341-355, 2011.

20. Heilbronn R and Weger S: Viral vectors for gene transfer: Current status of gene therapeutics. Handb Exp Pharmacol: 143-170, 2010.

21. Wang S, Zeng X, Liu Y, Liang C, Zhang H, Liu C, Du W and Zhang Z: Construction and characterization of a PDCD5 recombinant lentivirus vector and its expression in tumor cells. Oncol Rep 28: 91-98, 2012.

22. Robinson CJ and Stringer SE: The splice variants of vascular endothelial growth factor (VEGF) and their receptors. J Cell Sci 114: 853-865, 2001.

23. Ghosh S, Sullivan CA, Zerkowski MP, Molinaro AM, Rimm DL, Camp RL and Chung GG: High levels of vascular endothelial growth factor and its receptors (VEGFR-1, VEGFR-2, neuropilin-1) are associated with worse outcome in breast cancer. Hum Pathol 39: 1835-1843, 2008.

24. Yoshiji H, Gomez DE, Shibuya M and Thorgeirsson UP: Expression of vascular endothelial growth factor, its receptor, and other angiogenic factors in human breast cancer. Cancer Res 56: 2013-2016, 1996.

25. Goel HL and Mercurio AM: VEGF targets the tumour cell. Nat Rev Cancer 13: 871-882, 2013.

26. Guzmán-Hernández ML, Potter G, Egervári K, Kiss JZ and Balla T: Secretion of VEGF-165 has unique characteristics, including shedding from the plasma membrane. Mol Biol Cell 25: 1061-1072, 2014 
27. Kotterman MA and Schaffer DV: Engineering adeno-associated viruses for clinical gene therapy. Nat Rev Genet 15: 445-451, 2014.

28. Mahn M, Ron S and Yizhar O: Viral vector-based techniques for optogenetic modulation in vivo. In: Viral Vector Approaches in Neurobiology and Brain Diseases. Brambilla R (ed). Humana Press, Totowa, NJ, pp289-310, 2014.

29. Barrett DM, Singh N, Porter DL, Grupp SA and June CH: Chimeric antigen receptor therapy for cancer. Annu Rev Med 65: 333-347, 2013
30. Miller AD and Rosman GJ: Improved retroviral vectors for gene transfer and expression. Biotechniques 7: 980-990, 1989.

31. Maetzig T, Galla M,Baum C and Schambach A: Gammaretroviral vectors: Biology, technology and application. Viruses 3: 677-713, 2011.

32. Burns JC, Friedmann T, Driever W, Burrascano M and Yee JK Vesicular stomatitis virus $\mathrm{G}$ glycoprotein pseudotyped retroviral vectors: concentration to very high titer and efficient gene transfer into mammalian and nonmammalian cells. Proc Natl Acad Sci U S A 90: 8033-8037, 1993. 